\title{
Pratiques
}

Linguistique, littérature, didactique

183-184| 2019

oralité, littératie

\section{Les gestes à l'écrit dans les interactions numériques : description et fonctions}

\section{Pierre Halté}

\section{(2) OpenEdition}

\section{Journals}

Édition électronique

URL : http://journals.openedition.org/pratiques/7123

DOI : $10.4000 /$ pratiques.7123

ISSN : 2425-2042

Éditeur

Centre de recherche sur les médiations (CREM)

Référence électronique

Pierre Halté, "Les gestes à l'écrit dans les interactions numériques : description et fonctions ", Pratiques [En ligne], 183-184 | 2019, mis en ligne le 30 décembre 2019, consulté le 11 octobre 2020. URL : http://journals.openedition.org/pratiques/7123; DOI : https://doi.org/10.4000/pratiques.7123

Ce document a été généré automatiquement le 11 octobre 2020

(c) Tous droits réservés 


\title{
Les gestes à l'écrit dans les interactions numériques : description et fonctions
}

\author{
Pierre Halté
}

\section{Introduction}

1 Depuis qu'il est possible de communiquer de façon synchrone, à l'écrit, c'est-à-dire depuis les années 1970 (Dear, 2002), nous assistons à une inversion de la tendance jusqu'alors en vigueur dans l'histoire de la communication humaine : il ne s'agit plus de passer de l'oralité ${ }^{1}$ à la littératie (ce passage ayant été abondamment décrit, voir notamment Ong, 2002 [1982]), mais bien de la littératie à l'oralité. C'est tout naturel : nous redécouvrons depuis peu (voir McNeill, 2005) que notre langage, contrairement à ce que nos civilisations scripto-centrées préjugent, ne relève pas vraiment d'une combinaison entre différents systèmes sémiotiques, par exemple linguistique d'une part et mimo-gestuel d'autre part. Ce que l'on a trop longtemps considéré comme différents systèmes sont aujourd'hui plutôt conçus comme les facettes d'un seul processus communicatif.

«Gestes, langage et pensée sont maintenant vus comme différentes facettes d'un seul processus mental/cérébral/actionnel. Ils sont intégrés sur les plans actionnel, cognitif, et biologique. [Les gestes] sont conçus comme les ingrédients d'une dialectique image/langage qui sert de carburant au discours et à la pensée. » (McNeill, 2005, p. 3 nous traduisons)

Le régime de l'écrit, comme le montre W. J. Ong (2002 [1982]), nous a éloigné de cette conception, pourtant plausible - et fort argumentée - d'un point de vue scientifique et évolutionniste. L'écrit alphabétique (il en va, peut-être, autrement des écritures pictographiques), en tant que transcription de l'oral stabilisée et normée, donne en effet l'impression qu'il est possible de détacher le sens linguistique de tout ce qui, pourtant, participe à sa production : corps, voix, gestes, etc. Pour autant, dès lors que les premiers réseaux informatiques apparaissent (par exemple le réseau PLATO; Dear, 
2002), aux alentours de 1970, et qu'immédiatement sont inventés les premiers logiciels de t'chat, permettant de communiquer à l'écrit de façon synchrone, les utilisateurs inventent des façons de produire des sortes de "gestes à l'écrit »: des petites icônes, constituées de caractères de la banque $\mathrm{ASCII}^{2}$ superposés les uns aux autres, et représentant des mimiques faciales, des gestes, etc, que l'on nomme d'abord communément émoticônes, puis, plus récemment, émojis, entre autres dénominations ${ }^{3}$. Puisque cette technologie permet, pour la première fois, de simuler des interactions en «face à face » à l'écrit (Marcoccia, 2000), et puisque nos interactions naturelles, en face à face, reposent sur un système de communication global nécessitant, certes, notre langage parlé, mais aussi nos gestes et nos mimiques, il semble naturel que dans ce contexte apparaissent ces premières formes de " gestes à l'écrit ».

Depuis les années 1970, ces signes iconiques n'ont fait que progresser en termes de fréquence d'utilisation et sont présents non seulement dans les écrits numériques synchrones ( $t$ 'chat, messagerie mobile, par exemple) mais aussi asynchrones (forums, courriels, microblogging, etc.). Nous travaillerons, dans cet article, seulement sur les pictogrammes apparaissant dans les écrits numériques, qu'ils soient constitués de signes typographiques, comme :-) (à lire en penchant la tête vers la gauche), ou qu'ils soient plus figuratifs, comme $(-)$ ou 国相 $^{4}$. Il existe de nombreux autres phénomènes reposant sur l'iconicité, que nous laisserons de côté : stickers, gif animés, etc.

Cet article a deux objectifs : d'abord, proposer un cadrage permettant de conceptualiser la notion de "gestes à l'écrit » et de les classer en utilisant les typologies existantes; ensuite, décrire les usages et les fonctions de ces gestes à l'écrit.

Nous travaillerons sur un corpus utilisé au sein du projet ANR SoSweet (Magué, 2015), constitué d'un ensemble de plusieurs centaines de tweets sélectionnés parce qu'ils contiennent un ou plusieurs pictogrammes ${ }^{5}$. Ce choix nous permettra d'observer et de classer un certain nombre de phénomènes, mais il ne nous permettra pas de travailler sur le rôle des pictogrammes dans la dynamique conversationnelle, puisque nous n'avons pas accès, dans ce corpus, aux enchaînements de tours de parole qu'il est possible de voir sur Twitter, mais à des énoncés isolés. Par ailleurs, nous n'avons pas ici une perspective quantitative; il s'agit simplement d'observer différents usages de notre corpus, et non de rendre compte celui-ci de façon chiffrée. Nous nous consacrerons dans un premier temps à définir et à classer les gestes à l'écrit, puis, dans un second temps, nous nous intéresserons à leurs effets sur l'écrit ainsi qu'à leurs fonctions, aux niveaux syntaxiques, sémantiques et énonciatifs.

\section{I - Le geste à l'écrit?}

\section{1 - Gestes et discours}

6 La linguistique interactionnelle s'est attachée à établir une typologie des gestes, exploitable lors de l'analyse des interactions en face à face. L'une des typologies les plus employées (voir Azaoui, Saubesty \& Tellier, 2012) est celle proposée par D. McNeill (2005). Nous proposons de partir de cette typologie et d'y apporter quelques précisions d'ordre sémiotique, en nous appuyant notamment sur des remarques faites par K. M. Murphy (2003).

7 Commençons par préciser que la typologie gestuelle que nous convoquons ici concerne la gestualité intentionnelle. Il n'est pas question de traiter les gestes symptomatiques 
d'un état échappant au contrôle du sujet (tremblements, sursauts, etc.). Précisons aussi que nous incluons les mimiques faciales dans les gestes étudiés - là aussi, en nous cantonnant à celles qui relèvent d'une mise en scène consciente des affects (ce que J. Cosnier appelle « communication émotive "; Cosnier \& Vaysse, 1997) plutôt que d'un symptôme (qui relèverait, dans l'approche de J.Cosnier, de la «communication émotionnelle »).

8 La typologie élaborée par D. McNeill - qui ne se cantonne pas aux gestes indiquant des émotions, mais les inclut - inventorie quatre grands types de gestes :

La "gesticulation » est le mouvement qui incarne un sens relatif au discours qui l'accompagne. [...] La gesticulation est de loin le type de geste le plus fréquents en termes d'usage quotidien, et elle recouvre de nombreuses variantes et usages.

Les "gestes liés au discours" [speech-linked gestures] sont des parties des phrases elles-mêmes. Ces gestes occupent un espace grammatical dans une phrase [...], le geste complétant structurellement la phrase.

Les « emblèmes » [emblems] sont des signes conventionnels, comme le pouce levé ou le cercle (pouce et index qui se touchent alors que les autres doigts sont étendus) pour « OK».

La "pantomime" est un spectacle muet, un geste ou une séquence de gestes formant une ligne narrative, avec une histoire à raconter, produits sans discours. (McNeill, 2005, p. 5, nous traduisons)

9 À ce premier niveau de description des gestes, fondée sur leur relation avec le discours, s'ajoute en second niveau, une dimension sémiotique permettant de les classer selon la façon dont ils font sens. Ce second niveau s'appuie fortement sur la sémiotique peircienne, dont une des caractéristiques est de rendre compte de trois modes de relation entre signe et objet (voir Everaert-Desmedt, 1995) :

- L'iconicité, relation formelle dans laquelle le signe ressemble à l'objet (dessin, photographie, onomatopée) ;

- L'indexicalité, relation de contiguïté énonciative entre le signe et l'objet, dans laquelle le signe révèle l'existence de l'objet, ce dernier n'étant pas perceptible sans le signe (un geste de pointage, une empreinte dans le sable, mais aussi tous les déictiques de notre langue, sont des index);

- La relation symbolique, qui lie signe et objet par une convention adoptée par une communauté de locuteurs, sans autre motivation (la colombe pour la paix, le cœur pour l'amour, mais aussi la plupart des noms communs de la langue, comme « chien », etc.).

Partant de ces relations, trop brièvement décrites ici, D. McNeill propose, à un second niveau, quatre grandes catégories sémiotiques de gestes, qui peuvent se combiner avec les catégories du premier niveau: les gestes iconiques, métaphoriques, déictiques, et rythmiques.

Iconiques : ces gestes présentent des images d'entités concrètes et/ou d'actions. Ce sont des gestes dans lesquels la forme du geste et/ou son mode d'exécution incarne des aspects visuels de contenus sémantiques (ces aspects étant aussi présents dans le discours). [...]

Métaphoriques: les gestes peuvent aussi présenter des images de l'abstrait. Certains gestes impliquent un usage métaphorique de la forme : le locuteur apparaît comme s'il tenait un objet, comme s'il le présentait, mais le sens n'est pas de présenter un objet mais plutôt de tenir une « idée » ou un « souvenir » ou un autre «objet» abstrait dans sa main. Il y a alors une composante iconique (la forme du geste ressemble à la tenue d'un objet) et une composante métaphorique (tenir ou présenter quelque chose est une métaphore pour présenter un sens). 
Déictiques: bien que le geste déictique prototypique soit le pointage de l'index, n'importe quelle partie extensible du corps peut être utilisée pour pointer. Si les mains sont occupées, nous pouvons improviser un geste de pointage avec nos têtes, nez, coudes, pieds, ainsi qu'avec des abstractions comme l'« eidola » ou le regard de l'ophtalmologie antique. [...] Le pointage abstrait est une sorte de geste métaphorique, où l'espace et le lieu contenus sont utilisés pour créer un sens non spatial. [...] La deixis abstraite renverse la relation que le geste a avec son référent, par rapport au pointage concret. La deixis abstraite crée de nouvelles références dans l'espace ; la deixis concrète y trouve des références.

Rythmiques : ces gestes prennent la forme d'une main battant la mesure. (McNeill, 2005, p. 41, nous traduisons)

11 Le problème de ce classement sémiotique est qu'il laisse de côté la nature dynamique de la constitution du sens en contexte, en se cantonnant uniquement aux relations entre le geste et le discours. Or les relations peirciennes entre signes et objets ne sont pas à proprement parler des catégories mais des "potentialités ", aucun signe n'étant figé à priori dans un emploi donné 6 . C'est ce qu'illustre K. M. Murphy (2003) en critiquant un exemple proposé par D. McNeill (1992) : le geste de «balancier ». Ce geste, consistant à imiter une balance avec les mains (les paumes sont tournées vers le haut, et montent alternativement pour imiter les plateaux d'une balance), est analysé comme métaphorique par D. McNeill (1992) lorsqu'il accompagne un énoncé verbal contenant le verbe « décider ». Ainsi, le geste de balance est, pour D. McNeill, une métaphore de la décision en train de se prendre. K. M. Murphy précise cependant :

Ce que McNeill et ses collègues négligent dans cette typologie est que le geste luimême est iconique : il représente une balance, un objet du monde réel, tout comme un volant de voiture ou un livre. (Murphy, 2003, p. 34, nous traduisons)

12 En effet, avant d'être une métaphore de la décision, le geste de balancier est bien un geste iconique: il ressemble en premier lieu, formellement, à une balance. C'est ensuite, et en rapport avec ce qui est dit, qu'il prend une dimension symbolique (ou métaphorique), le concept de décision étant mis en rapport avec la référence à la balance par le locuteur.

13 Les catégories proposées par D. McNeill seront donc envisagées comme fondamentalement dynamiques, non exclusives les unes des autres. Par ailleurs, la plupart des approches peirciennes (voir les travaux de Fossali \& Dondero, 2011 [2006]) considèrent les relations entre signe et objet non pas comme des catégories fixes dans lesquels les signes doivent s'insérer, mais plutôt comme des potentialités toujours présentes dans n'importe quel signe, potentialités plus ou moins activées, et plus ou moins fortes, selon le contexte. Il est évident qu'un geste, comme n'importe quel énoncé, quel qu'il soit, a toujours, à un certain niveau, une dimension indexicale : sa production est nécessairement contigüe à une situation d'énonciation donnée, et la révèle par le fait même d'être énoncé. Par ailleurs, comme le montre K. M. Murphy dans l'exemple ci-dessus, un geste iconique peut aussi activer une relation symbolique avec un objet ; un geste iconique peut aussi être indexical, etc. Nous considèrerons donc que ces catégories ne sont pas « imperméables » mais doivent être prises comme un jeu dynamique et relationnel entre signe et objet.

\section{2 - À quelles conditions peut-on parler de " geste à l'écrit »?}

Trois niveaux d'analyse peuvent être convoqués pour tenter de répondre à cette question. À un premier niveau, celui de l'énonciation comme " acte ", toute production 
d'un énoncé, quel qu'il soit, relève d'un geste - que J. L. Austin (1970 [1962]) appelle, lorsque cela concerne la parole par exemple, " acte locutoire ». Ce premier niveau est d'ailleurs très bien circonscrit par A. Berrendonner lorsqu'il cherche à définir la notion d'acte :

Je résume : la définition du concept d'acte résulte des propositions suivantes :

1) Il existe un système sémiotique autonome dont les unités sont les gestes.

2) Un acte est un message construit selon les règles de ce code, muni d'une syntagmatique interne, et auquel est associé un résultat.

3) La production d'un énoncé verbal, envisagée comme événement purement gestuel, est un acte locutoire : l'acte d'énonciation. (Berrendonner, 1981, p. 83)

Il n'y a aucune raison de refuser ce statut " gestuel » au régime de l'écrit. Produire un énoncé écrit, que ce soit par l'intermédiaire d'un clavier et d'un écran d'ordinateur, d'un téléphone portable, ou d'un stylo et d'une feuille, est bien, fondamentalement, un geste. Par ailleurs, A. Berrendonner refuse que la notion d'acte soit employée autrement qu'en relation avec le système sémiotique mimo-gestuel: selon lui, les notions d'actes illocutoires et perlocutoires ne sont pas pertinentes puisqu'elles relèvent de tout autre chose que du geste :

Parler, c'est tout autre chose que faire : c'est remplacer l'acte par quelque chose qui n'est pas un acte, mais peut en provoquer les effets. Lorsque Jill énonce «J'ai faim », elle utilise le langage comme un bon moyen de ne pas agir ; elle évite ainsi d'avoir à accomplir des gestes, c'est-à-dire cueillir la pomme qu'elle convoite. Mais l'acte, c'est, selon Bloomfield, Jack qui l'accomplit en grimpant à l'arbre. Jill, hormis " quelques petits mouvements de la langue et des lèvres ", n'a, elle, accompli aucun acte digne de ce nom. (Berrendonner, 1981, p. 80)

Néanmoins, A. Berrendonner ne rejette pas les notions d'illocutoire ou de perlocutoire. Simplement, il refuse d'inscrire ces concepts dans le champ de l'acte, qui, pour lui, est avant tout intrinsèquement lié au système sémiotique mimo-gestuel. En d'autres termes, ce n'est pas parce que parler peut avoir les mêmes effets que faire, que ces deux procès ont le même statut. Nous souscrivons globalement à cette analyse, avec, malgré tout, une objection : il existe bien, dans la langue elle-même, des mots dont le sens et les effets sont uniquement liés à leur production effective, par exemple, les interjections ${ }^{7}$. Lorsque je produis une interjection, comme «Aïe!», je ne décris pas un état de fait, cette description entraînant par inférences un effet perlocutoire; je ne produis pas un mot dont la sémantique référentielle renverrait à un concept présupposant un acte illocutoire (comme « demander» ou « remercier»); je performe une sorte de cri conventionnel, dont le sens même est intrinsèquement lié à l'acte locutoire de profération de l'interjection. Or, les interjections sont dans le dictionnaire ; elles relèvent bien du "parler ", elles font partie de la langue - C. Olivier invente d'ailleurs, pour les nommer, le terme de «cri parlé » (Olivier, 1986). Les interjections, existent évidemment à l'écrit. Cette objection ouvre un second niveau d'analyse : audelà de l'acte locutoire, qui est "vraiment» un geste, il y a, dans les énoncés linguistiques eux-mêmes, des éléments dont le sens repose sur leur « performance »qu'elle soit écrite ou parlée. Par ailleurs, comme nous l'avons défendu (Halté, 2018a), c'est le cas aussi pour les émoticônes que l'on peut qualifier d'«interjections graphiques » et dont il va être question ici.

17 Enfin, à un troisième et dernier niveau d'analyse, lié au second comme nous le verrons, nous constatons que nos écrits numériques contiennent, de façon massive, des icônes visuels de gestes : pictogrammes, stickers, gifs, etc., qui ressemblent, dans leur forme, à 
des gestes ou à des mimiques faciales produites lors d'interactions à l'oral. Ces icônes ont, généralement, le sens des gestes auxquelles elles ressemblent :

(1) Un grand 矂 @XXXX vous avez grave assuré toute vos chorégraphies vous êtes

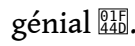

La relation aux gestes, incorporés à l'écrit, est ici évidente; il reste cependant à savoir si ces signes peuvent être considérés comme appartenant au régime de l'écrit ou s'il s'agit d'un système sémiotique tout à fait différent, se combinant avec le régime de l'écrit. L'intégration à ce dernier est très difficile à défendre pour ce qui est des gifs (petites vidéos qui s'insèrent plus ou moins bien dans les énoncés), des stickers (images de grande taille apparaissant parfois dans les tchats ou les forums) car il s'agit dans ces deux cas, manifestement, d'un système sémiotique tout à fait autre que celui de l'écriture, pour des raisons spatiales, mais aussi sémantiques et syntaxiques. Nous n'étudierons pas ici ces phénomènes. Point important: il est évident que certains pictogrammes, représentant des gestes de façon iconiques, comme (-) ou 迎地, sont, de par leur caractère d'icônes, directement et intuitivement liés aux gestes auxquels ils ressemblent. Néanmoins, nous considèrerons, dans ce qui suit, que toute production de pictogramme, même lorsque ce dernier ne représente pas un geste utilisé habituellement à l'oral, constitue un geste à l'écrit (correspondant au minimum à un geste iconique dans la typologie de D. McNeill). Ainsi nous considérons que produire un pictogramme de voiture, comme ${ }_{6959}^{619}$, en relation avec un discours écrit, constitue une performance qui revient, d'un point de vue sémiotique, à effectuer, grâce au système mimo-gestuel, une imitation d'une voiture (par exemple en manipulant un volant imaginaire) en relation avec un discours oral. Cette position s'appuie sur les arguments développés ci-avant.

Il est possible d'argumenter fortement en faveur de l'intégration de ces signes au régime de l'écrit. Ce sont en effet des pictogrammes, terme dont l'étymologie en dit long : pictus, « image » + gramma, « écrit». Ce sont des signes hybrides par excellence, comme ne manque pas de le remarquer P. Vaillant (2013):

Les pictogrammes ont ceci de particulier qu'ils sont à la fois des images et des textes, ou, pour parler techniquement, que leur mode de fonctionnement sémiotique tient en partie à celui des signes iconiques, et en partie à celui des textes des langues naturelles écrites. [...] Un pictogramme est un signe qui voudrait se faire comprendre selon le principe iconique - c'est-à-dire, idéalement, par reconnaissance visuelle primaire, sans qu'un code appris préalablement soit nécessaire à cette reconnaissance, mais qui, par ailleurs, s'inscrit dans un système sémiotique organisé, à l'extrême un système d'écriture. (Vaillant, 2013)

Par système d'écriture, P. Vaillant entend d'une part "combinaison d'un axe syntagmatique et paradigmatique » et, d'autre part, "système de signes ». Les pictogrammes ont en effet pour caractéristique - et c'est le cas dans les écritures pictographiques - de pouvoir se combiner sur un axe syntagmatique pour former une ligne narrative, mais aussi de pouvoir commuter les uns avec les autres sur un axe paradigmatique, ce qui implique des contraintes spatiales, de formes, de stylisation... Les signes que nous étudions ici, qu'ils soient constitués de signes typographiques, comme:-), ou des formes graphiquement plus figuratives, comme $(-)$, répondent exactement à cette définition: leur interprétation repose sur une saisie iconique visuelle, ils font partie d'un système de signes, reposant sur des combinaisons de "sous-signes" que nous analyserons plus loin, ils se combinent sur un axe syntagmatique (qu'ils partagent avec l'écrit alphabétique), et ils peuvent commuter 
entre eux sur un axe paradigmatique. Ces icônes de gestes sont donc des pictogrammes, ce qui est un argument fort pour les rattacher à la fois au régime de l'écrit et au système mimo-gestuel. un concept présent dans le discours verbal. Plus complexe encore, le pictogramme employé peut à la fois avoir une valeur iconique, ET une valeur indexicale portant sur l'objet représenté :

(4) Bonne lecture ! (Très beau chat t...)

i, le chat, qualifié de "très beau», est représenté de façon iconique par le pictogramme ; mais ses yeux, en forme de cœur, indiquent que le locuteur aime le chat ainsi représenté! Ce pictogramme est donc à la fois icône d'une tête de chat, et index de l'émotion du locuteur. commun, mais parfois un verbe) par un pictogramme, ce qui correspond, dans la typologie de D. McNeill, aux gestes "liés au discours» (" speech linked gestures»). Le 
pictogramme prend alors la place d'un élément de la chaîne syntaxique, tout comme le ferait un geste iconique dans une interaction orale :

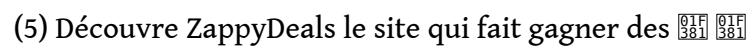

Lorsque le ou les pictogrammes remplacent un nom commun, ils constituent alors des équivalents de gestes iconiques. Notons que dans l'exemple ci-dessus, le pluriel attendu, "cadeaux », est rendu perceptible par la répétition du pictogramme : il y a plusieurs cadeaux.

Usage moins fréquent, les pictogrammes peuvent prendre la place d'un verbe :

(6) Okay je me lève malade, un mal de crâne pas possible, je bosse jusqu'à $18 \mathrm{~h}$ (au

lieu de $20 \mathrm{~h} \mathrm{mdr)} \mathrm{et} \mathrm{j'organise} \mathrm{une} \mathrm{soirée} \mathrm{chez} \mathrm{moi.} \mathrm{Je} \mathrm{(:).}$

L'analyse est ici plus complexe et doit avoir recours à plusieurs éléments de la typologie de D. McNeill. Il s'agit bien d'une icône de mimique faciale. Cette mimique faciale indique une émotion, mais elle est employée comme le serait un verbe, renvoyant sémantiquement à un procès (que l'on pourrait gloser par " pleurer ", par exemple). Il s'agirait donc bien ici, au premier niveau de la typologie, d'un "speech linked gesture " que l'on pourrait classer, au second de niveau de la typologie, dans les «gestes métaphoriques ", actualisant en même temps des composantes iconiques et indexicales. Nous trouvons aussi dans les corpus des icônes de gestes "emblèmes ", conventionnels, comme le pouce levé, le signe « $\mathrm{OK}$ » formé en joignant le pouce et l'index et en tendant les autres doigts, ou encore les mains jointes en prière :

(7) $23 \mathrm{~h}$ et on est toujours pas sortie. Vous le sentez le décompte dans le métro? ? [916

Dans cet exemple, le geste de "pouce levé » est aussi gesticulatoire : il porte sur ce qui est décrit dans la partie verbale de l'énoncé. Autre exemple de geste à l'écrit « emblème" :

(8) Possible de me follow stp 㽧????

Ici, les mains sont jointes dans un geste conventionnel qui sert à appuyer la demande faite verbalement.

Enfin, nous trouvons aussi, dans les corpus numériques, des pantomimes, c'est-à-dire des séquences de gestes à l'écrit utilisé dans une courte chaîne narrative :

(9) Une belle idée cadeau ? Le livre Sport \& Cinéma avec un large et superbe

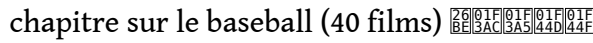

$\mathrm{Si}$, dans cet exemple, la suite de pictogrammes peut aussi être considérée comme gesticulatoire, puisqu'elle est en interaction manifeste avec le contenu du discours verbal, il n'en reste pas moins que la séquence elle-même relève de la pantomime : il s'agit bien de mimer une séquence narrative, allant du spectacle " cinématographique " de baseball aux applaudissements du spectateur. On trouve par ailleurs de pures séquences de gestes, utilisées sans discours verbal, dans certaines formes de salutations reproduisant les pratiques des locuteurs dans les interactions réelles :

(10) 路国新

37 Ici, il s'agit de reproduire une séquence de gestes, utilisée dans certaines communautés pour saluer son interlocuteur, consistant en un contact claqué entre les mains des deux interlocuteurs, suivi d'un contact entre leurs poings fermés (parfois appelé « check»).

L'utilisation des pictogrammes dans les écrits numériques recouvre donc tous les gestes décrits par D. McNeill pour les interactions orales (à l'exception des gestes «rythmiques», ne pouvant exister qu'à l'oral pour des raisons de superposition 
possible des canaux vocal et gestuel, impossible à l'écrit) et ce dans les deux niveaux de sa typologie : au premier niveau, fonctionnel, on trouve des gestes à l'écrit relevant de la gesticulation, du speech linked gesture, du geste emblème, ainsi que de la pantomime ; au second niveau, sémiotique, on trouve des gestes aux dimensions indexicales, iconiques et symboliques/métaphoriques, dimensions parfois actualisées simultanément dans des dynamiques parfois complexes. Il reste maintenant à explorer les fonctions et les effets qu'ont ces gestes sur l'écrit verbal, aux niveaux sémantique, énonciatif, syntaxique et, plus généralement, interactionnel.

\section{II - Fonctions et effets des gestes à l'écrit}

\section{1 - Effets syntaxiques}

Les gestes à l'écrit n'ont pas d'incidence syntaxique à proprement parler: ils ne modifient pas les mots de la phrase se situant dans leur entourage. Néanmoins, ils s'intègrent de différentes façons à la chaîne syntaxique, posent des questions quant à leur rapport à la ponctuation, et posent aussi des problèmes de spatialisation de l'écrit. Le cas le plus simple est celui des "gestes liés au discours" (" speech linked gestures »). Dans ce cas de figure, les pictogrammes prennent tout simplement la place des unités syntaxiques qu'ils remplacent, tout comme le font les gestes gesticulatoires iconiques à l'oral :

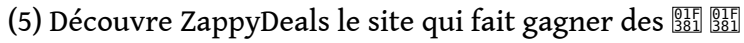

(6) Okay je me lève malade, un mal de crâne pas possible, je bosse jusqu'à $18 \mathrm{~h}$ (au

lieu de $20 \mathrm{~h} \mathrm{mdr}$ ) et j'organise une soirée chez moi. Je (:).

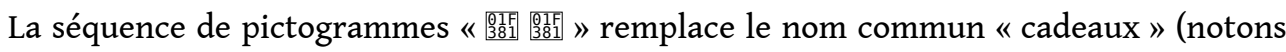
au passage que le pluriel est signalé par la production de plusieurs pictogrammes), et le pictogramme " (:) " remplace un verbe. Ce remplacement lexical ne peut être interprété que grâce à l'entourage syntaxique immédiat: le déterminant "des " précède nécessairement un nom commun, et le pronom personnel «je ", nécessairement un verbe, ce qui force, dans l'exemple (6), à attribuer au pictogramme une valeur de procès qu'il n'aurait pas nécessairement s'il n'était pas précédé de « je ». Notons enfin que rien n'empêche, dans le cas de pictogramme(s) employé(s) pour remplacer un nom commun, de leur accoler un adjectif (comme, par exemple, on pourrait le faire avec l'adjectif « beaux » dans (5)).

Dans les autres cas de figures, à savoir les gestes à l'écrit relevant de la gesticulation (qu'ils soient déictiques - lorsqu'ils indiquent l'affect du locuteur - ou iconiques lorsqu'ils illustrent le contenu sémantique de la partie écrite de l'énoncé) et ceux relevant de la pantomime, l'intégration à la chaîne syntaxique pose des problèmes différents. En effet, la différence majeure, en ce qui concerne la catégorie "gesticulation", entre les gestes à l'écrit et les gestes dans les interactions orales, est qu'un geste gesticulatoire, dans une interaction orale, peut parfaitement se superposer à la chaîne discursive : il s'agit de deux canaux de communications parallèles. À l'écrit, évidemment, cela est impossible : il n'y a plus qu'un seul canal, et il faut donc placer les gestes quelque part sur une chaîne syntaxique matérialisée par l'écrit.

Se posent donc les questions du rapport des gestes à l'écrit à la ponctuation et de leur rôle dans la segmentation syntaxique des énoncés écrits. Ces questions seront plus largement explorées dans un article à venir, mais nous pouvons néanmoins en tracer ici 
les contours. Les pictogrammes relevant de la gesticulation peuvent être positionnés à gauche de la partie écrite du tour de parole, à sa droite (c'est le cas le plus fréquent), ou enfin en son incise. Cela est notamment lié à des questions de portée (sémantique plutôt que syntaxique) qui ont été décrites par ailleurs (Halté, 2017). Dans le cas des pictogrammes antéposés, la question du remplacement de la ponctuation, ou du positionnement par rapport à cette dernière, ne se pose pas (du moins en français). Par contre, lorsque les pictogrammes sont en incise ou en fin du tour de parole, ils peuvent :

- remplacer le signe de ponctuation (souvent une virgule, un point-virgule, voire des points de suspension)

(12) "Une vierge scandaleuse scan scan scan scan scandaleuse" ss aussi vierge $:$ j'aime trop cette chanson

- être placés avant le signe de ponctuation

(2) J'ai trop mangé chez ma pote, encore plus qu'hier $\&$...et elle m'a fait deux tuperware blindés pour chez moi

- être placés après le signe de ponctuation

(11) La fille qui lance son café sans mettre le gobelet dans la machine... :-:Elle

arrête pas de gueuler et moi j'en peux plus de rire! :-:

Les effets de ces différents positionnements concernent le calcul de la portée sémantique du pictogramme gesticulatoire. Le fait de placer ce pictogramme à gauche du signe de ponctuation, surtout lorsque c'est en incise du tour de parole, indique clairement que le pictogramme doit être compris comme portant sur le contenu sémantique énoncé à sa gauche, comme par exemple dans (2). Il peut aussi s'agir pour le locuteur de vouloir combiner, dans un certain ordre, les modalités portées par la ponctuation et celles portées par le pictogramme. Ainsi, dans (11), la modalité exclamative précède la modalité appréciative portée par ": : "; changer l'ordre d'apparition de ces deux signes modifierait cette interprétation, le rire étant alors pris dans la portée de la modalité exclamative signifiée par le point d'exclamation.

Il arrive que le pictogramme apparaisse à l'endroit où l'écrit traditionnel nécessiterait un signe de ponctuation. Difficile de dire alors s'il s'agit d'un véritable remplacement, le pictogramme occupant à la fois les fonctions de modalisation et de segmentation, tenues habituellement par le signe de ponctuation, ou simplement d'un effet collatéral, les pictogrammes gesticulatoires ne pouvant se placer qu'après ou avant des clauses syntaxiques complètes et " finies ", mais jamais, par exemple, entre un déterminant et un nom commun, tout comme la plupart des signes de ponctuation.

\section{2 - Fonctions « sémantiques »}

Les fonctions sémantiques des gestes à l'écrit s'appuient en partie sur les relations entre texte et image, bien analysées par J.-M. Klinkenberg (2008).

Dans le cas des speech-linked gestures, où le pictogramme remplace un mot de la chaîne syntaxique, sa fonction sémantique est de renvoyer, de façon iconique, à un objet. Là où le nom commun « cadeau » renvoie - selon l'approche sémantique que l'on privilégie à une classe d'entités, ou constitue une représentation symbolique composée d'un ensemble de sèmes, le pictogramme ${ }_{3011}^{111}$ ressemble, dans sa forme, à un objet, et c'est cette ressemblance visuelle qui en fonde le sens. Le fonctionnement sémantique d'un signe iconique est bien décrit par U. Eco (1978 [1975]), mais aussi par le Groupe $\mu$ (1992), nous ne nous y attardons donc pas. Nous signalons néanmoins que si cette sémantique 
permet de bien décrire les pictogrammes représentant des objets, il reste certainement un travail à faire concernant les pictogrammes susceptibles de signifier des procès, comme par exemple « (:) » dans (6), où le pictogramme prend la place du verbe. Notons aussi la valeur sémantique déclenchée par la répétition d'un pictogramme, notamment dans ce dernier cas de figure où il est placé à la place d'un verbe :

(12) @X @YJJ moi je vous :; :; :; (:), c'est vendredi je suis heureux d'être en weekend $:$ :

En général, la répétition des pictogrammes indique une intensification du procès signifié, qui pourrait être d'ailleurs indiquée par un adverbe d'intensification («je vous embrasse fort »).

Pour ce qui relève de la gesticulation à l'écrit, on trouve donc deux cas de figures. Dans le premier, les pictogrammes jouent un rôle d'illustration de la partie verbale de l'énoncé. Dans le second, ils font porter l'affect ou l'attitude du locuteur sur la partie verbale de l'énoncé. Les trois types de relations sémantiques mises au jour par J.-M. Klinkenberg (redondance, différence, opposition entre contenu sémantique verbal et iconique) peuvent théoriquement s'appliquer dans ces deux cas.

Pour les pictogrammes gesticulatoires illustratifs, on ne trouve dans notre corpus quasiment que des relations de redondance, ce qui s'explique assez facilement : il s'agit pour la plupart des utilisateurs de rendre leurs énoncés plus pertinents, et non pas d'en brouiller l'interprétation en opposant texte et image. Ces relations de redondance peuvent reposer sur des connotations. C'est le cas, par exemple, dans (3):

(3) joue les pères noël et vous accompagne jusqu'au réveillon! [814

On peut considérer ici que ce à quoi renvoie l'icône de sapin est une connotation commune aux noms communs « réveillon » et " père noël ». Notons aussi que dans les relations de redondance, les pictogrammes sont souvent utilisés comme "surenchère " par rapport au contenu sémantique véhiculé par la partie verbale de l'énoncé, notamment par la répétition du pictogramme :

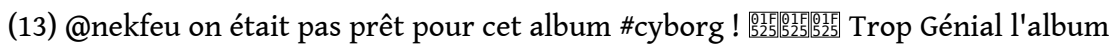

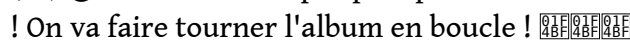

51 Le contenu sémantique «faire tourner l'album en boucle » est repris par la succession des pictogrammes de disques.

Dans le second cas de figure, les gestes à l'écrit font porter l'affect ou l'attitude du locuteur sur le contenu énoncé dans la partie écrite. Toutes les relations sémantiques décrites par J.-M. Klinkenberg sont, dans ce cas, possible: redondance, différence, opposition, chacune provoquant des inférences responsables d'interprétations variées. La redondance est le type de relation le plus fréquent, comme par exemple pour le dernier pictogramme de (11):

(11) La fille qui lance son café sans mettre le gobelet dans la machine... :-:Elle

arrête pas de gueuler et moi j'en peux plus de rire! :-:

Mais parfois, le contenu sémantique verbal et gestuel n'ont pas de lien apparent, comme dans (2) :

(2) J'ai trop mangé chez ma pote, encore plus qu'hier $\curvearrowright *$ \&..et elle m'a fait deux

tuperware blindés pour chez moi

Ce qui est décrit, le fait d'avoir trop mangé, n'a rien à voir avec ce qui est signifié par les pictogrammes représentant le locuteur en train de pleurer. Cette différence déclenche, comme décrit par exemple dans la théorie de la pertinence de D. Sperber \& D. Wilson (1989), un calcul inférentiel forçant à faire un lien entre les contenus 
sémantiques des parties iconiques et verbales de l'énoncé, et aboutissant à une reconstitution d'un contenu sémantique global que l'on peut gloser de la sorte: «le locuteur est triste d'avoir trop mangé ».

Enfin, dernière possibilité, les pictogrammes gesticulatoires déictiques peuvent s'opposer au contenu sémantique signifié par la partie verbale de l'énoncé, conduisant la plupart du temps à une interprétation ironique ou, à tout le moins, relevant de la plaisanterie :

(14) Restons modestes,juste de gros connards :-: :) !!!

Ces oppositions peuvent aussi servir à construire une menace, par exemple.

\section{3 - Fonctions énonciatives}

Parmi l'ensemble des pictogrammes étudiés et classés précédemment, certains ont un rôle de marqueur énonciatif (ou "marqueur discursif», terme utilisé par Dostie \& Pusch, 2007). Il s'agit des pictogrammes déictiques gesticulatoires, qui servent à indiquer l'affect, ou plus généralement l'attitude du locuteur, pour le ou la faire porter sur le contenu sémantique de la partie verbale de l'énoncé. Nous reproduisons ici les quelques points qui définissent les marqueurs discursifs :

- Les MD appartiennent aux classes mineures et ils sont morphologiquement invariables.

- Ils ne contribuent pas au contenu propositionnel des énoncés et c'est pourquoi leur présence ou leur absence ne modifie pas la valeur de vérité des énoncés auxquels ils sont joints.

- Ils ont tendance à constituer des unités prosodiques indépendantes, si bien qu'ils sont en général extérieurs à la structure de la phrase.

- Ils sont optionnels sur le plan syntaxique, c'est-à-dire que, dans les cas où ils sont joints à un énoncé, leur absence n'entraine pas une agrammaticalité. De plus, ils n'entrent pas dans une structure argumentale et ils peuvent occuper différentes positions par rapport à un énoncé, s'ils ne sont pas utilisés comme mots-phrases.

- Ils jouent un rôle au-delà de la phrase et ils relèvent de la macro-syntaxe du discours (Blanche-Benveniste 1997). (Dostie et Pusch, 2007, p. 3)

Le terme de marqueur discursif est utilisé en linguistique pour désigner des unités de langue. Nous proposons d'étendre cette catégorie à tous les signes, y compris nonverbaux, dont la fonction est d'indiquer, hic et nunc, une relation entre le locuteur et un ou plusieurs éléments de la situation d'énonciation. Considérons par exemple (2) :

(2) J'ai trop mangé chez ma pote, encore plus qu'hier $\&$...et elle m'a fait deux tuperware blindés pour chez moi

Ces émoticônes portent sur l'énoncé situé à leur gauche. Elles servent à indiquer l'attitude du locuteur concernant le contenu propositionnel énoncé précédemment: elles n'en modifient pas les conditions de vérité et n'ont pas d'incidence syntaxique. Elles seraient par ailleurs tout à fait commutables avec des interjections - ou d'autres marqueurs discursifs - indiquant des modalités affectives négatives. Nous avons argumenté ailleurs (Halté, 2018a) en faveur d'un rapprochement, sur un plan énonciatif, entre les interjections et les pictogrammes déictiques; il nous semble que la catégorie des marqueurs discursifs convient parfaitement pour décrire leurs rôles énonciatifs. Les pictogrammes index d'affect - dont la fonction est d'indiquer l'émotion $\mathrm{du}$ locuteur- que nous appelons émoticônes, ne contribuent pas au contenu propositionnel des énoncés; elles sont optionnelles sur le plan syntaxique et, nous l'avons vu, occupent différentes positions par rapport à l'énoncé; elles relèvent 
globalement de la macro-syntaxe. Le critère prosodique n'est évidemment pas pertinent pour rendre compte des pictogrammes comme marqueurs discursifs.

Ces pictogrammes remplissent deux fonctions énonciatives qui sont liées entre elles. En tant que déictiques indiquant l'affect du locuteur, elles contribuent tout d'abord à la construction de ce dernier - rappelons que, dans la typologie des instances énonciatives d'O. Ducrot, le locuteur est l'instance, construite par et dans l'énoncé, qui prend en charge (au sens de : Rabatel, 2009 ou Laurendeau, 2009) la vérité ou ON-vérité de son contenu (si l'on ajoute les objections de Berrendonner, 1981). Les émoticônes ne peuvent pas être utilisées pour référer aux affects de quelqu'un d'autre que « celui qui écrit ». On n'en trouve d'ailleurs pas au sein de discours rapportés (voir Halté, 2018b). L'identification de l'instance prenant en charge le contenu propositionnel de l'énoncé est possible, en partie, grâce aux émoticônes, qui sont autant d'indices ou traces de la construction de cette instance.

Leur seconde fonction majeure est de modaliser les propositions énoncées, à la suite desquelles elles apparaissent. Certains constituants des émoticônes (que nous appelons « iconèmes »; voir Halté, 2019), comme les formes représentant les bouches ou les yeux, ont pour fonction de signifier des modalités (au sens de Gosselin, 2010), le plus souvent affectives, mais aussi, éventuellement, épistémiques (comme une émoticône de surprise : (:) ou déontiques (comme certaines icônes de geste de refus: fonction est facilement démontrée par des tests de commutation (voir Halté, 2018a) pour une analyse plus détaillée). Observons par exemple (2'), dans lequel nous avons modifié les émoticônes :

(2') J'ai trop mangé chez ma pote, encore plus qu'hier :-):-...et elle m'a fait deux tuperware blindés pour chez moi

61 L'interprétation change : là où, en (2), le locuteur indique qu'il est triste d'avoir « trop mangé chez sa pote ", en (2'), il indique qu'il en est content. Le contenu propositionnel ne change pas, mais l'attitude modale est contraire. Cette modalisation peut être monologique, c'est-à-dire porter sur un énoncé produit par le locuteur - comme dans (2) -, mais elle peut aussi être dialogique, c'est-à-dire porter sur un énoncé déjà modalisé par l'interlocuteur - dans le cadre de conversations via t'chat par exemple (Halté, 2016). Par ailleurs, cette modalisation peut avoir plusieurs visées : le contenu propositionnel, mais aussi la relation à l'interlocuteur ou encore la forme que prend l'énoncé8. L'emploi de ces gestes à visée énonciative, à l'écrit, est tout à fait similaire à celui qui est fait des gestes gesticulatoires déictiques à l'oral, à ceci près que la modalité repose sur un calcul de portée qui est plus simple à l'oral, puisque discours et geste peuvent être produits en même temps.

\section{Conclusion}

62 Avec les pictogrammes, les éléments mimo-gestuels de notre communication dans les interactions à l'oral font irruption dans le régime de l'écrit. Nous pouvons qualifier ces signes de gestes à l'écrit: non seulement parce que leur sens repose sur leur "performance ", comme c'est d'ailleurs le cas pour des éléments linguistiques comme les interjections, mais aussi et surtout parce qu'il s'agit de reproductions iconiques de gestes, fonctionnant en système et susceptibles de commuter sur l'axe paradigmatique et de se combiner sur l'axe syntagmatique. De plus, on peut classer ces pictogrammes en suivant les typologies élaborées pour rendre compte des gestes à l'oral (par exemple, 
celle de McNeill, 2005). L'insertion de ces éléments dans le régime de l'écrit pose néanmoins des questions différentes. Puisqu'il est impossible, comme à l'oral, de prononcer un énoncé en même temps qu'on produit un geste, les gestes à l'écrit doivent se positionner par rapport à une chaîne syntaxique linéaire, dont ils sont à priori exclus : rejetés à sa gauche, ou, le plus souvent, à sa droite ; parfois, inséré entre deux clauses syntaxiques. Cela a des conséquences, notamment sur la ponctuation et la segmentation des énoncés. Sur le plan sémantique, par contre, ces gestes à l'écrit fonctionnent tout à fait comme le font les gestes de l'oral, ou les images interagissant avec le texte. Enfin, sur un plan énonciatif, les similitudes sont elles aussi nombreuses : ils participent à la construction d'un locuteur, en permettant la prise en charge de contenu; ils servent à modaliser des propositions. Les gestes, jusqu'alors traditionnellement et abusivement cantonnés au monde de l'oralité, n'y sont donc plus limités. La tendance générale allant vers une multiplicité de plus en plus forte des systèmes sémiotiques (texte, image, vidéo, son, interfaces, etc.) constituant nos communications numériques, nous pouvons nous attendre à ce que d'autres catégories, structurant la façon dont nous percevons notre propre communication, soient prochainement bouleversées. En l'état actuel des choses, il reste de nombreux points à explorer concernant les relations entre pictogrammes et texte, qui démontrent toute la complexité multimodale de la construction du sens en interaction. Comment rendre compte, par exemple, de l'association de séquences de pictogrammes représentant des gestes et des objets, pour signifier un procès, le tout étant lié à une formule de salutation, comme dans (15)?

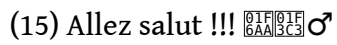

\section{BIBLIOGRAPHIE}

AUSTIN, J. L. (1970) [1962]. Quand dire, c'est faire. Trad. de l'anglais G. Lane. Paris : Éditions du Seuil.

AZAOUI, B., SAUBESTY, J. \& TELLIER, M. (2012). « Segmentation et annotation du geste : Méthodologie pour travailler en équipe ».JEP-TALN-RECITAL 2012. Atelier DEGELS 2012 (Défi GEste Langue des Signes). Grenoble, p. 41-55. En ligne : https://hal.archives-ouvertes.fr/hal-01510435.

BERRENDONNER, A. (1981). Éléments de pragmatique linguistique. Paris : Éditions de Minuit.

COSNIER, J., \& VAYSSE, J. (1997). « Sémiotique des gestes communicatifs ». Nouveaux actes sémiotiques 52 , p. 7-28.

DEAR, B. L. (2002). «Emoticons and Smileys on PLATO in the 1970s ». Plato people. Consulté

31 janvier 2019. En ligne : http://www.platopeople.com/emoticons.html.

Dostie, G., \& PUSCH, C. D. (2007). « Présentation. Les marqueurs discursifs. Sens et variation ».

Langue française 154, p. 3-12.

ECO, U. (1978) [1975]. Trattato di semiotica generale. Milano : Bompiani.

EVERAERT-DESMEDT, N. (1995). Le processus interprétatif. Liège : Éditions Mardaga. 
FOSSALI, P. B., DONDERO, M. G. (2011) [2006]. Sémiotique de la photographie. Trad. de l'italien par N. Roelens, S. D'Arconso \& C. Lévy. Limoges : Presses universitaires de Limoges et du Limousin. GOSSELIN, L. (2010). Les modalités en français. La validation des représentations. Amsterdam/New York : Rodopi.

Groupe $\mu$ (1992). Traité du signe visuel. Pour une rhétorique de l'image. Paris : Éditions du Seuil.

HALTÉ, P. (2016). « Émoticônes et modalisation dans un corpus d'enseignement par t'chat ». Études de linguistique appliquée. Revue de didactologie et de lexiculturologie des langues-cultures 184, p. 441-453.

HALTÉ, P. (2017). « Positionnement syntaxique des interjections et des émoticônes : modalisation, portée, visée ». Les cahiers de praxématique 69. En ligne : https://halshs.archives-ouvertes.fr/ halshs-01803669.

HALTÉ, P. (2018a). Les émoticônes et les interjections dans le tchat. Limoges : Lambert-Lucas.

HALTÉ, P. (2018b). « Émoticône et modalisation : ancrage énonciatif du locuteur dans un corpus de t'chat ». In : Nita, R. \& Valetopoulos, F. (dirs). L'expression des sentiments. De l'analyse linguistique aux applications. Rennes : Presses universitaires de Rennes, p. 185-201. En ligne : https:// halshs.archives-ouvertes.fr/halshs-01814688.

HALTÉ, P. (2019), « Iconicité et signification modale : l'émoticône, de l'icône du corps au geste énonciatif », MEI. Médiation et information 47, p. 143-157.

HALTÉ, P. (2020). « Emojis, émoticônes, smileys ? Proposition de classement terminologique selon des critères énonciatifs ». Interfaces numériques 8 (2), p. 365-385. En ligne : https://www.unilim.fr/ interfaces-numeriques/3956.

KLINKENBERG, J.-M. (2008). « La relation texte-image. Essai de grammaire générale ». Bulletin de la Classe des Lettres et des sciences morales et politiques. $6^{\mathrm{e}}$ série, t. XIX. Bruxelles : Académie Royale des Sciences, des Lettres et des Beaux-Arts, pp. 21-79. En ligne : https://fr.scribd.com/document/ 56692073/La-relation-texte-image-Essai-de-grammaire-generale-J-M-Klinkenberg.

LAURENDEAU, P. (2009). « Préassertion, réassertion, désassertion : construction et déconstruction de l'opération de prise en charge ». Langue française 162, p. 55-70.

LHOTE, É. (2001). « Pour une didactologie de l'oralité ». Études de linguistique appliquée. Revue de didactologie et de lexiculturologie des langues-cultures 123-124, p. 445-453.

MARCOCCIA, M. (2000). « La représentation du nonverbal dans la communication écrite médiatisée par ordinateur ». Communication et organisation 18. En ligne : https://journals.openedition.org/ communicationorganisation/2431.

MCNEILL, D. (1992). Hand and Mind. What Gestures Reveal about Thought. Chicago : University of Chicago Press.

MCNEILL, D. (2005). Gesture and Thought. Chicago : University of Chicago Press.

MURPHY, K. M. (2003). « Building Meaning in Interaction: Rethinking Gesture Classifications ». Crossroads of Language, Interaction, and Culture 5, p. 29-47.

OLIVIER, C. (1986). Traitement pragmatique des interjections en français. Thèse de doctorat en linguistique à Toulouse 2. En ligne : http://www.theses.fr/1986TOU20029 ONG, W. J. (2002) [1982].Orality and Literacy. The Technologizing of the Word. Londres/New York: Routledge. 
PERRIN, L. (2008). « Le sens montré n'est pas dit ». In : Birkelund, M., Hansen, M.-B. \& Norén, C. (dirs). L'énonciation dans tous ses états. Mélanges offerts à Henning Nølke à l'occasion de ses soixante ans. Berne/Berlin.Bruxelles : Peter Lang, p. 157-187.

RABATEL, A. (2009). « Prise en charge et imputation, ou la prise en charge à responsabilité

limitée... ». Langue française 162, p. 71-87.

SPERBER, D., \& WILSON, D. (1989). La pertinence. Communication et cognition. Paris : Éditions de Minuit.

VAILlant, P. (2013). « Sémiologie des pictogrammes ». Texto! Textes \& Cultures XVIII (4). En ligne : http://www.revue-texto.net/index.php?id=3336.

\section{NOTES}

1. Par « oralité », nous entendons :

«[...] une mise en acte d'un long processus psycho-socio-linguistico-physiologique qui s'accompagne d'une émission et/ou d'une réception sonore faisant usage d'organes vocaux et auditifs. Il s'agit bien d'un passage à l'acte, de nature physique et physiologique, qui s'appuie sur la voix que tout individu a construite au cours de son apprentissage du langage. Quand on parle de mise en acte, on suppose une phase préparatoire au cours de laquelle un certain nombre de facteurs sont sollicités. » (Lhote, 2001, § 15)

2. American Standardized Code for Information Interchange, premier système d'encodage informatique des caractères typographiques (128 caractères à l'origine).

3. Pour plus de précisions terminologiques, voir Halté (2020). Nous utiliserons dans cet article majoritairement deux termes: celui de pictogramme, terme le plus général, pour désigner (comme le propose Vaillant, 2013) des signes dont l'interprétation repose sur leur iconicité, et qui font partie d'un système d'écriture (ils résultent d'une combinatoire de "sous-signes" et s'organisent sur les axes paradigmatique et syntagmatique); et celui d'émoticône, qui désignera des pictogrammes indiquant un affect du locuteur ou plus généralement une attitude (Halté 2018a).

4. Pour des définitions plus précises de ces signes et des arguments les constituant comme une seule et même catégorie, voir Halté (2018).

5. Les tweets sont produits à partir de trente-quatre clients différents (dont les cinq plus fréquents sont Twitter for iPhone, Twitter for Android, Twitter Web Client, TweetDeck et Twitter for iPad). Étant donné la diversité des modes de présentation des tweets selon le logiciel utilisé, nous avons délibérément opté pour une mise en forme de nos exemples qui ne préserve que leur contenu linguistique, sans essayer de mimer l'interface d'un logiciel arbitrairement sélectionné. L'interface n'a de toute façon pas d'incidence sur ce que nous essayons de décrire ici. La graphie d'origine a été conservée.

6. Chez S. C. Peirce, c'est la notion d'habitus qui fige les signes dans un emploi donné. Ce sont des usages multiples et répétés, au sein d'une communauté de locuteurs, qui installent un signe dans une relation habituelle d'iconicité, d'indexicalité ou dans une relation symbolique. Il est cependant toujours possible de changer volontairement cette relation. Nous en verrons des exemples lorsque nous aborderons la sémiose de certains pictogrammes. 
7. L. Perrin (2008) étend cette objection à d'autres éléments linguistiques: verbes performatifs, adverbes d'énonciation, Pour plus de détails concernant cette approche des interjections, voir notamment Perrin (2008), Halté (2018).

8. Pour plus de détails à ce sujet, voir Halté, 2018a.

\section{RÉSUMÉS}

Nous nous intéressons dans cette étude aux pictogrammes (émoticônes, émojis) faisant partie des énoncés produits dans les écrits numériques médiés, plus particulièrement dans les tweets. Notre hypothèse est que ces pictogrammes relèvent du "geste à l'écrit ", contribuant à l'hybridité, entre oralité et littératie, de ces discours. Cet article a deux objectifs : d'abord, proposer un cadrage sémiotique permettant de conceptualiser la notion de "gestes à l'écrit " et de les classer en utilisant les typologies existantes; ensuite, décrire les usages et les fonctions de ces "gestes à l'écrit ». Nous proposons, dans un premier temps, une réflexion sur les rapports entre geste et discours, puis nous confrontons ces pictogrammes à la typologie gestuelle de D. McNeill (1992; 2005), fondée sur certains aspects de la sémiotique peircienne, pour les classer. Dans un second temps, nous étudions les effets et les fonctions de ces gestes à l'écrit, aux niveaux syntaxique, sémantique et énonciatif.

This article is about pictograms (emoticons, emojis) in computer mediated writings, such as tweets. Our hypothesis is that these pictograms are literally "written gestures", adding to the orality/literacy hybridity of those discourses. This study has two purposes: first, to build the notion of "written gestures" and to be able to categorize and classify them according to existing typologies; then, to describe the uses and the pragmatic functions of these "written gestures". First, we deal with the links between speech and gestures, and we use D. McNeill's $(1992 ; 2005)$ gestures typology, which use peircian semiotics at its core, to see if the pictograms we study fit in it; then, we study the effects and pragmatic functions of those written gestures at the syntactic, semantic and discursive level.

\section{INDEX}

Mots-clés : émoticônes, émojis, geste à l'écrit, écrits numériques, gestualité et discours Keywords : emoticons, emojis, written gestures, computer mediated writings, gestures and speech

\section{AUTEUR}

\section{PIERRE HALTÉ}

Université de Paris-Descartes, EDA, F-75006 Paris, France 